\title{
Entropy dimension and variational principle
}

\author{
by \\ Young-Ho Ahn (Mokpo), Dou Dou (Nanjing) and \\ KYEWON KoH PARK (Suwon)
}

\begin{abstract}
Recently the notions of entropy dimension for topological and measurable dynamical systems were introduced in order to study the complexity of zero entropy systems. We exhibit a class of strictly ergodic models whose topological entropy dimensions range from zero to one and whose measure-theoretic entropy dimensions are identically zero. Hence entropy dimension does not obey the variational principle.
\end{abstract}

1. Introduction. Entropy is an isomorphism invariant which measures the exponential growth rate of complexity of dynamical systems. It is a complete invariant for the class of Bernoulli transformations. It is also well known that any measurable dynamical system with positive entropy is isomorphic to a skew product with the Bernoulli transformation of the same entropy.

Zero entropy systems cover a wide class of transformations exhibiting different "random" behaviors. Interval exchange transformations have entropy zero. Simple maps in joining theory, which include the Chacon transformation, irrational rotations and horocycle flows, also have zero entropy [G]. Indeed, the set of zero entropy systems is a dense $G_{\delta}$ subset of all measure preserving transformations. To classify the "randomness" or "complexity" of zero entropy systems, we need some new invariants which are finer than entropy $[\mathrm{F}, \mathrm{KT}]$. For example, the notions of entropy dimension and entropy dimension set are introduced to help the understanding of the various levels of complexity of zero entropy systems [DHP1, DHP2]. We can briefly say that entropy dimension measures the subexponential growth rate of orbits, and the entropy dimension set measures the complexity of factors. The topological or measure-theoretic entropy dimension of a translation on a compact group is zero. Also if a dynamical system has positive entropy, then its entropy dimension is one. It is shown that for any given $0<\alpha<1$, there exists a transformation whose entropy dimension is $\alpha[\mathrm{FP}$. It has been our hope

2010 Mathematics Subject Classification: Primary 37A35; Secondary 37A25.

Key words and phrases: entropy dimension, variational principle, zero entropy system. 
that many of the properties of entropy will be generalized and strengthened to entropy dimensions. This would lead to a better understanding of zero entropy systems. For example, under very mild conditions, two topological or measurable dynamical systems with different entropy dimension sets are disjoint in the sense of Furstenberg [DHP1, DHP2].

Let $X$ be a compact metric space and $T$ be a homeomorphism on $X$. The variational principle for the classical entropy says that the topological entropy of the dynamical system $(X, T)$ is the supremum of all measuretheoretic entropies of $T$. Just as in the case of entropy, it is shown in [DHP2] that metric entropy dimensions are always less than or equal to the topological entropy dimension. And there are examples where metric entropy dimension is the same as the topological entropy dimension [DHP2]. A natural question is whether the topological entropy dimension of a system is the supremum of the measurable entropy dimensions over all probability invariant measures. We call this property the variational principle for entropy dimension.

It is fairly easy to construct topological examples of given topological entropy dimension. However, many of these examples, in particular the physical models, have no physically meaningful finite invariant measures but $\sigma$-finite invariant measures. In this paper, we consider a class $\left\{X_{\alpha}\right\}$ of symbolic dynamical systems, where $X_{\alpha}$ is generated by a special infinite sequence $\mathbf{u}_{\alpha}$. This system is known to be minimal and its topological entropy dimension is $\alpha[\mathrm{C}$. We show that these topological dynamical systems are uniquely ergodic and their measure-theoretic entropy dimensions are identically zero. Hence these examples reveal differences between topological and measurable entropy dimensions. They show that the variational principle does not hold for entropy dimension. In fact these are the only known examples yet of fractional topological entropy dimensions which have finite invariant measures.

2. Preliminaries. There are several equivalent definitions of classical entropy for topological and measurable dynamical systems. Motivated by one of those definitions, the notions of entropy dimensions have been introduced [DHP1, DHP2, FP]. Henceforth, by entropy dimension we mean measure-theoretic entropy dimension.

2.1. Entropy dimension. We recall the definition of topological entropy dimension.

Let $(X, T)$ be a topological dynamical system and $\mathcal{C}_{X}^{o}$ be the set of all finite open covers of the compact metric space $X$. For $0 \leq \alpha \leq 1$, we consider two measures of complexity of the topological dynamical system for $\mathcal{U} \in \mathcal{C}_{X}^{o}$ : 


$$
\begin{aligned}
& \bar{D}(T, \alpha, \mathcal{U})=\limsup _{n \rightarrow \infty} \frac{\log \mathcal{N}\left(\bigvee_{i=0}^{n-1} T^{-i} \mathcal{U}\right)}{n^{\alpha}}, \\
& \underline{D}(T, \alpha, \mathcal{U})=\liminf _{n \rightarrow \infty} \frac{\log \mathcal{N}\left(\bigvee_{i=0}^{n-1} T^{-i} \mathcal{U}\right)}{n^{\alpha}},
\end{aligned}
$$

where $\mathcal{N}(\mathcal{U})$ is the smallest cardinality of subcovers of $\mathcal{U}$.

We define $\bar{D}(T, \mathcal{U})=\sup \{0 \leq \alpha \leq 1: \bar{D}(T, \alpha, \mathcal{U})>0\}$, and the upper topological entropy dimension $\bar{D}(X, T)$ as the supremum of $\bar{D}(T, \mathcal{U})$ over all finite open covers $\mathcal{U}$.

Similarly, we define $\underline{D}(T, \mathcal{U})=\sup \{0 \leq \alpha \leq 1: \underline{D}(T, \alpha, \mathcal{U})>0\}$ and the lower topological entropy dimension $\underline{D}(X, T)$ as the supremum of $\underline{D}(T, \mathcal{U})$ over all finite open covers $\mathcal{U}$. It is known that if $\mathcal{U}$ is a generating open cover (i.e. $\left.\lim _{n \rightarrow \infty} \operatorname{diam}\left(\bigvee_{i=0}^{n-1} T^{-i} \mathcal{U}\right)=0\right)$, then $\bar{D}(X, T)=\bar{D}(T, \mathcal{U})$ and $\underline{D}(X, T)=\underline{D}(T, \mathcal{U})$ respectively. If $\bar{D}(X, T)=\underline{D}(X, T)$, then we call it the topological entropy dimension of $(X, T)$.

Let $T$ be a measure preserving transformation on a standard Borel probability space $(X, \mathcal{B}, \mu)$, and $\mathcal{P}$ be a finite measurable partition of $X$. An analogous definition of entropy dimension would be

$$
\bar{D}_{\mu}^{H}(\mathcal{P})=\sup \left\{0 \leq \alpha \leq 1: \limsup _{n \rightarrow \infty} \frac{1}{n^{\alpha}} H\left(\bigvee_{i=0}^{n-1} T^{-i} \mathcal{P}\right)>0\right\} .
$$

However it was shown that $\sup _{\mathcal{P}} \bar{D}_{\mu}^{H}(\mathcal{P})=1$ if $\bar{D}_{\mu}^{H}(\mathcal{Q})>0$ for some partition $\mathcal{Q}$, hence this is not an isomorphism invariant [FP]. We need to modify the topological definition and define entropy dimension by counting the number of $\bar{d}$-balls of names $\mathrm{R}$.

Let $\mathcal{P}=\left\{P_{1}, \ldots, P_{k}\right\}$ be a given partition and let $\mathcal{P}_{[0, n)}(x)$ denote the first $n$-name of the atom $P_{[0, n)}(x) \in \bigvee_{i=0}^{n-1} T^{-i} \mathcal{P}$. That is, $\mathcal{P}_{[0, n)}(x)=$ $x_{0} x_{1} \cdots x_{n-1}$ where $x_{i}=l$ if $T^{i}(x) \in P_{l}$ for all $0 \leq i<n$. For a point $x \in X$, we define

$$
b(x, n, \epsilon)=\left\{y \in X: \bar{d}\left(\mathcal{P}_{[0, n)}(x), \mathcal{P}_{[0, n)}(y)\right)<\epsilon\right\},
$$

where $\bar{d}$ is the Hamming metric defined by

$$
\bar{d}\left(x_{0} x_{1} \cdots x_{n-1}, y_{0} y_{1} \cdots y_{n-1}\right)=\frac{1}{n} \#\left\{i: x_{i} \neq y_{i} \text { for } 0 \leq i<n\right\} .
$$

Let $K(n, \epsilon)$ be the smallest number $K$ such that there exists a subset of $X$ of measure at least $1-\epsilon$ covered by $K$ balls of type $b(x, n, \epsilon)$. We define

$$
\begin{aligned}
& \bar{D}_{\mu}(\mathcal{P}, \epsilon)=\sup \left\{0 \leq \alpha \leq 1: \limsup _{n \rightarrow \infty} \frac{\log K(n, \epsilon)}{n^{\alpha}}>0\right\}, \\
& \underline{D}_{\mu}(\mathcal{P}, \epsilon)=\sup \left\{0 \leq \alpha \leq 1: \liminf _{n \rightarrow \infty} \frac{\log K(n, \epsilon)}{n^{\alpha}}>0\right\} .
\end{aligned}
$$

Set $\bar{D}_{\mu}(\mathcal{P})=\lim _{\epsilon \rightarrow 0} \bar{D}_{\mu}(\mathcal{P}, \epsilon)$ and $\underline{D}_{\mu}(\mathcal{P})=\lim _{\epsilon \rightarrow 0} \underline{D}_{\mu}(\mathcal{P}, \epsilon)$. 
We define the upper entropy dimension $\bar{D}_{\mu}(X, T)$ as the supremum of $\bar{D}_{\mu}(\mathcal{P})$ and the lower entropy dimension $\underline{D}_{\mu}(X, T)$ as the supremum of $\underline{D}_{\mu}(\mathcal{P})$ over all finite measurable partitions $\mathcal{P}$ respectively.

Similarly to the case of topological dynamical systems, if the two values are equal, then we call it the entropy dimension of $(X, \mathcal{B}, \mu, T)$, denoted by $D_{\mu}(X, T)$. It is easy to show that if $\mathcal{P}$ is a generating partition, then $\bar{D}_{\mu}(X, T)=\bar{D}_{\mu}(\mathcal{P})$ and $\underline{D}_{\mu}(X, T)=\underline{D}_{\mu}(\mathcal{P})$.

2.2. Cassaigne's example. Cassaigne constructed a uniformly recurrent $0-1$ sequence which has superpolynomial but subexponential growth rate of the complexity function [C]. In this subsection, we briefly recall his construction.

We recall that the dyadic valuation word $\mathbf{v}=v_{1} v_{2} v_{3} \ldots$ is an infinite word with $v_{n}$ being the dyadic valuation of $n$, i.e. the largest integer $h$ such that $2^{h}$ divides $n$. This word can be described in the following way. Let $z_{1}=0$ and define a sequence of finite words by the recursive relation $z_{j+1}=z_{j} j z_{j}$. Then the dyadic valuation word $\mathbf{v}$ is the limit of the sequence of words $\left(z_{j}\right)$ $(j \geq 1)$. More precisely, $z_{2}=z_{1} 1 z_{1}=010, z_{3}=z_{2} 2 z_{2}=0102010$ and

$$
\mathbf{v}=\lim _{j \rightarrow \infty} z_{j+1}=0102010301020104 \cdots .
$$

By using the dyadic valuation word and substituting each $j(j=0,1,2, \ldots)$ by a proper finite word of 0's and 1's, a sequence which has superpolynomial but subexponential growth rate of $n$-blocks can be constructed.

Let $\varphi: \mathbb{R}^{+} \rightarrow \mathbb{R}^{+}$be a function such that

(i) $\varphi(t) \gg \log t$,

(ii) $\varphi$ is differentiable, except possibly at 0 ,

(iii) $\varphi^{\prime}(t) \ll t^{-\beta}$ for some positive constant $\beta$,

(iv) $\varphi^{\prime}$ is decreasing.

For example, $\varphi(t)=t^{1 / 2}$ and $\varphi(t)=t^{1 / 3} \log (t+5)$ satisfy all the above conditions.

Given any finite or infinite set $A$, we denote by $A^{*}$ the collection of finite or infinite words over $A$. Define inductively the substitution $\psi: \mathbb{N}^{*} \rightarrow\{0,1\}^{*}$ and the family $\left(x_{k}\right)_{k \in \mathbb{N}}$ of prefixes of the dyadic valuation word $\mathbf{v}$ as follows:

(a) $\psi(0)=0, \psi(1)=1$;

(b) $x_{k}$ is the longest prefix of $\mathbf{v}$ such that

$$
\left|\psi\left(x_{k}\right)\right| \leq \max \left(\varphi^{-1}(k+1)-\varphi^{-1}(k)-1,0\right) ;
$$

(c) for all $j \geq 1$,

$$
\psi(2 j)=\psi\left(x_{\lfloor\log j\rfloor}\right) 0 \psi(j) \quad \text { and } \quad \psi(2 j+1)=\psi\left(x_{\lfloor\log j\rfloor}\right) 1 \psi(j),
$$

where $\lfloor a\rfloor$ denotes the maximal integer not exceeding $a$. 
Let $\mathbf{u}=\psi(\mathbf{v})$.

In the case of $\varphi(t)=t^{1 / 2}$, by the condition (b) above, we have $\varphi^{-1}(t)=t^{2}$ and $x_{k}$ is the longest prefix of $\mathbf{v}$ such that $\left|\psi\left(x_{k}\right)\right| \leq 2 k$. The following gives the first few terms of $\mathbf{u}$.

- $x_{0}=\varepsilon, \psi\left(x_{0}\right)=\varepsilon$ where $\varepsilon$ denotes the empty word;

- since $\psi(0)=0, \psi(1)=1$ and $\mathbf{v}=010 \cdots$, we have $x_{1}=01$ and $\psi\left(x_{1}\right)=01$

- since $\psi(2)=\psi\left(x_{0}\right) 0 \psi(1)=01, \psi(3)=\psi\left(x_{0}\right) 1 \psi(1)=11, \psi(4)=$ $\psi\left(x_{1}\right) 0 \psi(2)=01001$, we have

$$
\begin{aligned}
\mathbf{u} & =\psi\left(z_{3} 3 z_{3} 4 \cdots\right)=\psi(0102010301020104 \cdots) \\
& =01001010110100101001001 \cdots
\end{aligned}
$$

The word $\mathbf{u}$ has the following properties (see [C, Theorem 3]).

Proposition 2.1. $\mathbf{u}$ is a uniformly recurrent word and its complexity satisfies

$$
\log p_{\mathbf{u}}(n) \sim \varphi(n),
$$

where $p_{\mathbf{u}}(n)$ is the number of blocks of length $n$ in $\mathbf{u}$.

Let $X_{\mathbf{u}}$ be the set of all bi-infinite sequences $x \in\{0,1\}^{\mathbb{Z}}$ such that every $n$-block of $x$ is an $n$-block of $\mathbf{u}$. Since $\mathbf{u}$ is uniformly recurrent, $\left(X_{\mathbf{u}}, \sigma\right)$ is a minimal topological dynamical system where $\sigma$ is the shift map. In this paper, we restrict our attention to the sequences $\mathbf{u}_{\alpha}$ which are induced by the functions $\left\{\varphi(t)=t^{\alpha}, 0<\alpha<1\right\}$ which satisfy all Cassaigne's conditions.

For the existence and uniqueness of an invariant measure of the system $\left(X_{\mathbf{u}}, \sigma\right)$, we need to compute the frequency of blocks in a sufficiently long word $w$. For these computations we will show that there exists $k$ such that $\psi\left(z_{k}\right)$ makes up most of the word $w$ (Lemma 3.4). We also need to investigate the structures of $\psi\left(z_{k}\right)$ and $\psi(i)$ together with their lengths. We need the following estimates.

Lemma 2.2 ([C, Lemma 3]). The sequence $(|\psi(i)|)$ is nondecreasing. Moreover, for $i \geq 1,|\psi(i)|$ depends only on $\lfloor\log i\rfloor$ and $1+\varphi^{-1}(\lfloor\log i\rfloor)-\lfloor\log i\rfloor\left(1+\varphi^{-1}(\lfloor\log \lfloor\log i\rfloor\rfloor)\right) \leq|\psi(i)| \leq 1+\varphi^{-1}(\lfloor\log i\rfloor)$.

We need to consider the occurrences of $n$-blocks in $\psi\left(z_{k}\right)$, since $\psi\left(z_{k}\right)$ 's make up most of $\mathbf{u}$. To count the occurrences, we also need the following lemma which describes the relationship between the length of $\psi\left(z_{k}\right)$ and $k$.

Lemma 2.3. Let $n \geq 3$ and $g(n)=\max \left\{i:\left|\psi\left(z_{i+1}\right)\right| \leq n-2\right\}$. Then there exists a constant $c$ such that $\log (n-2)-c<g(n) \leq \log (n-2)$.

Proof. By the conditions on $\varphi(t)$, we have $\varphi(n) \gg \log n$ and $n \gg$ $\varphi^{-1}(\lfloor\log n\rfloor)$. So there exists $k>0$ such that $\varphi^{-1}(\lfloor\log n\rfloor) \leq n+k-1$ for all $n$. Let $\left|\psi\left(z_{n}\right)\right|=a_{n}$. Since $|\psi(n)| \leq 1+\varphi^{-1}(\lfloor\log n\rfloor)$ and $\left|\psi\left(z_{n+1}\right)\right|=$ 
$2\left|\psi\left(z_{n}\right)\right|+|\psi(n)|$, we have $2 a_{n} \leq a_{n+1} \leq 2 a_{n}+n+k$ with $a_{1}=1$. Let $b_{n}$ be the sequence defined by the recursive relation $b_{n+1}=2 b_{n}+n+k$ with $b_{1}=1$. Then $a_{n} \leq b_{n}$ and

$$
b_{n}=\frac{k+3}{2} 2^{n}-(n+1)-k \leq \frac{k+3}{2} 2^{n} .
$$

Let $c$ be a constant such that $\frac{k+3}{2} \leq 2^{c-2}$. Then $2^{n-1} \leq a_{n} \leq 2^{n+c-2}$. Hence

$$
2^{g(n)} \leq\left|\psi\left(z_{g(n)+1}\right)\right| \leq 2^{g(n)+c-1}, \quad 2^{g(n)+1} \leq\left|\psi\left(z_{g(n)+2}\right)\right| \leq 2^{g(n)+c} .
$$

Therefore $2^{g(n)} \leq n-2$ and $2^{g(n)+c}>n-2$. Hence $g(n) \leq \log (n-2)$ and $g(n)>\log (n-2)-c$.

3. Unique ergodicity of the symbolic system $X_{\mathbf{u}_{\alpha}}$. It is well known that there is a symbolic dynamical system which is minimal but not uniquely ergodic (see, for example, [WS]). In this section, we will prove that each symbolic system $X_{\mathbf{u}_{\alpha}}$ is uniquely ergodic. First, we will show that $X_{\mathbf{u}_{\alpha}}$ has an invariant measure $\mu_{\alpha}$ for which $\mathbf{u}_{\alpha}$ is generic. If there is no confusion, we will denote $\mu_{\alpha}$ by $\mu, \mathbf{u}_{\alpha}$ by $\mathbf{u}$ and $X_{\mathbf{u}_{\alpha}}$ by $X_{\alpha}$ for notational convenience.

Proposition 3.1. For each symbolic system $X_{\alpha}$, there exists an invariant measure $\mu_{\alpha}$ for which $\mathbf{u}_{\alpha}$ is generic.

Proof. Let $f_{j}^{B}$ be the frequency of the block $B$ in $\psi\left(z_{j}\right)$. Let $g_{i}$ be the number of occurrences of $B$ in $\psi(i)$, and $k(i)$ be the number of occurrences of $B$ which intersect both $\psi\left(z_{i}\right)$ and $\psi(i)$. Since the number of occurrences of $i$ in $z_{j}$ is $2^{j-i-1}$, the length of $\psi\left(z_{j}\right)$ is $\sum_{i=0}^{j-1} 2^{j-i-1}|\psi(i)|$ and

$$
f_{j}^{B}=\frac{\sum_{i=0}^{j-1} 2^{j-i-1}\left(g_{i}+k(i)\right)}{\sum_{i=0}^{j-1} 2^{j-i-1}|\psi(i)|}=\frac{\sum_{i=0}^{j-1} 2^{-i}\left(g_{i}+k(i)\right)}{\sum_{i=0}^{j-1} 2^{-i}|\psi(i)|} .
$$

Since the growth rate of $|\psi(i)|$ is subexponential, and since $g_{i} \leq|\psi(i)|$ and $k(i) \leq 2|B|, \sum_{i=0}^{j-i} 2^{-i}|\psi(i)|$ and $\sum_{i=0}^{j-i} 2^{-i}\left(g_{i}+k(i)\right)$ converge as $j$ goes to infinity. So the sequence $f_{j}^{B}$ converges to a constant $f^{B}$. Hence for given $\epsilon$, there exists $J$ such that $\left|f_{j}^{B}-f^{B}\right|<\epsilon / 2$ for all $j \geq J$. Now we can find $N$ such that for all $n \geq N, \mathbf{u}_{1}^{n}=u_{1} \cdots u_{n}$ is covered by $\left\{\psi\left(z_{j}\right): j \geq J\right\}$ except for $n \epsilon / 2$ coordinates. Thus the frequency of $B$ in $u_{1} \cdots u_{n}$ is $f^{B} \pm \epsilon$ for all $n \geq N$, which implies the existence of an invariant measure $\mu_{\alpha}$.

Now we will prove the unique ergodicity of $X_{\alpha}$. The following classical result is taken from Theorem 4.9 of [G] (see also [W]).

TheOREM 3.2. A topological dynamical system $(X, T)$ is uniquely ergodic iff for every continuous function $g \in C(X)$ the sequence of functions $n^{-1} \sum_{i=0}^{n-1} g \circ T^{i}$ converges pointwise to a constant function. 
For the unique ergodicity of shift spaces, since the finite blocks make up a dense collection of clopen sets for shift spaces, it is enough to check that the characteristic function of the form $f=\mathbf{1}_{B}$ for a finite block $B$ satisfies the conclusion of the previous theorem. To prove the unique ergodicity, we define the following notion.

Definition 3.3. Let $v$ be a block of length $k$ and $w$ be a block of length $n \geq k$. Suppose $w=w_{1} \cdots w_{n}$ where $w_{i}=0$ or 1 . We define

$$
d(v, w)=\frac{k}{n} \#\left\{i_{l}: w_{i_{l}} w_{i_{l+1}} \cdots w_{i_{l+k}}=v, 1 \leq i_{l} \leq n-k, i_{l+1} \geq i_{l}+k\right\} .
$$

That is, $d(v, w)$ is the maximum value of the ratios of nonoverlapping parts of $w$ covered by $v$. We call $d(v, w)$ the density of $v$ in $w$.

Lemma 3.4. For any $\epsilon>0$, there exists $K=K(\epsilon)$ such that for any $k \geq K$, there is $N=N(\epsilon, k)$ such that $d\left(\psi\left(z_{k}\right), w\right)>1-\epsilon$ for any subword $w$ of $\mathbf{u}$ with length greater than $N$.

Proof. For given $\epsilon>0$, there exists $K=K(\epsilon)$ such that

$$
\frac{\sum_{i=K}^{\infty}|\psi(i)| 2^{-i}}{\sum_{i=0}^{K}|\psi(i)| 2^{-i}}<\frac{\epsilon}{32} .
$$

Due to the construction of the infinite word $\mathbf{u}$, we prove the lemma by the following steps.

Step 1. Let $w$ be the prefix of $\mathbf{u}$ of length $n$. Then there is a unique $l$ such that $\left|\psi\left(z_{l}\right)\right| \leq n<\left|\psi\left(z_{l+1}\right)\right|$. Hence $w$ is a prefix of $\psi\left(z_{l+1}\right)$, and $\psi\left(z_{l}\right)$ is a prefix of $w$. When $l \geq k$,

$$
\begin{aligned}
d\left(\psi\left(z_{k}\right), w\right) & \geq 1-\frac{\sum_{i=k}^{l}|\psi(i)| 2^{l-i}+\left|\psi\left(z_{k}\right)\right|}{n} \\
& \geq 1-\frac{\sum_{i=k}^{l}|\psi(i)| 2^{l-i}+\left|\psi\left(z_{k}\right)\right|}{\left|\psi\left(z_{l}\right)\right|} \\
& =1-\frac{\sum_{i=k}^{l}|\psi(i)| 2^{l-i}+\left|\psi\left(z_{k}\right)\right|}{\sum_{i=0}^{l-1}|\psi(i)| 2^{l-i-1}} \\
& =1-2 \frac{\sum_{i=k}^{l}|\psi(i)| 2^{-i}}{\sum_{i=0}^{l-1}|\psi(i)| 2^{-i}}-\frac{\left|\psi\left(z_{k}\right)\right|}{\sum_{i=0}^{l-1}|\psi(i)| 2^{l-i-1}} .
\end{aligned}
$$

So there exists $N_{1}=N_{1}(\epsilon, k)$ such that

$$
d\left(\psi\left(z_{k}\right), w\right)>1-\epsilon / 8 \quad \text { for }|w|>N_{1}(\epsilon, k),
$$

since the dyadic valuation word has the recursive relation $z_{j+1}=z_{j} j z_{j}$.

If $w$ is a suffix of some $\psi\left(z_{l+1}\right)$ and $\psi\left(z_{l}\right)$ is a suffix of $w$, then for $|w|>N_{1}$, we get $d\left(\psi\left(z_{k}\right), w\right)>1-\epsilon / 8$ by the same argument. 
Step 2. From the construction of $\mathbf{u}$, one has

$$
\psi(m)=\psi\left(x_{\lfloor\log m\rfloor-1}\right) e \psi\left(x_{\lfloor\log m\rfloor-2}\right) e \cdots,
$$

where $e$ can be 0 or 1 . When $\left|\psi\left(x_{i}\right)\right|>N_{1}$, by Step 1 ,

$$
d\left(\psi\left(z_{k}\right), \psi\left(x_{i}\right)\right)>1-\epsilon / 8 .
$$

Let $i_{0}$ be the smallest integer $i$ such that $\left|\psi\left(x_{i}\right)\right|>N_{1}$. Then

$$
d\left(\psi\left(z_{k}\right), \psi(m)\right)>1-\frac{\frac{\epsilon}{8} \sum_{i=i_{0}}^{\lfloor\log m\rfloor-1}\left|\psi\left(x_{i}\right)\right|+\lfloor\log m\rfloor+\sum_{i=0}^{i_{0}-1}\left|\psi\left(x_{i}\right)\right|}{|\psi(m)|} .
$$

By Lemma 2.2, we have $|\psi(m)| \gg \log m$. Hence there exists $N_{2}=$ $N_{2}(\epsilon, k)$ such that

$$
\frac{\lfloor\log m\rfloor+\sum_{i=0}^{i_{0}-1}\left|\psi\left(x_{i}\right)\right|}{|\psi(m)|}<\frac{\epsilon}{8} \quad \text { for all } m>N_{2} .
$$

Thus $d\left(\psi\left(z_{k}\right), \psi(m)\right)>1-\epsilon / 4$ for all $|w|=|\psi(m)|>\left|\psi\left(N_{2}\right)\right|$.

If $w$ is a suffix of some $\psi(m)$, then without loss of generality we may assume that $m$ is the smallest integer such that $w$ is a suffix of $\psi(m)$. Since $\varphi(t)=t^{\alpha}, 0<\alpha<1$, for given $\epsilon$ there exists $N_{3}(\epsilon, k)$ such that $\left|\psi\left(x_{\lfloor\log m\rfloor-1}\right)\right| / \psi(\lfloor m / 2\rfloor)<\epsilon / 8$ for all $m>N_{3}$. Let $N_{4}=\left|\psi\left(N_{2}\right)\right|+\left|\psi\left(N_{3}\right)\right|$. Since

$$
\begin{aligned}
& d\left(\psi\left(z_{k}\right), w\right) \\
& \quad>1-\frac{\frac{\epsilon}{8} \sum_{i=i_{0}}^{\lfloor\log m\rfloor-2}\left|\psi\left(x_{i}\right)\right|+\left|\psi\left(x_{\lfloor\log m\rfloor-1}\right)\right|+\lfloor\log m\rfloor+\sum_{i=0}^{i_{0}-1}\left|\psi\left(x_{i}\right)\right|}{|\psi(\lfloor m / 2\rfloor)|},
\end{aligned}
$$

we have $d\left(\psi\left(z_{k}\right), w\right)>1-3 \epsilon / 8$ for $|w|>N_{4}$.

Note that for given $w$, there exists a unique $m^{\prime}$ such that $\left|\psi\left(m^{\prime}\right)\right| \leq$ $|w|<\left|\psi\left(m^{\prime}+1\right)\right|$, and if $|w|>\left|\psi\left(N_{2}\right)\right|$, then $\left(\left\lfloor\log m^{\prime}\right\rfloor+1\right) /\left|\psi\left(m^{\prime}\right)\right|<\epsilon / 8$. We may also assume that $N_{1} /\left|\psi\left(N_{2}\right)\right|<\epsilon / 8$.

Suppose $w$ is a prefix of some $\psi(m)$. Then

$$
w=\psi\left(x_{\lfloor\log m\rfloor-1}\right) e \psi\left(x_{\lfloor\log m\rfloor-2}\right) e \cdots r
$$

where $r$ is a prefix of $\psi\left(x_{j}\right)$ for some $j$. Then the cardinality of the random part in $w$, i.e. the number of appearances of $e$ in $w$, is at most $\left\lfloor\log m^{\prime}\right\rfloor+1$. Hence if $|w|>\left|\psi\left(N_{2}\right)\right|$, then

$d\left(\psi\left(z_{k}\right), w\right)>1-\frac{\epsilon}{8}-\frac{\left\lfloor\log m^{\prime}\right\rfloor+1}{|w|}-\frac{N_{1}}{|w|}>1-\frac{\epsilon}{8}-\frac{\left\lfloor\log m^{\prime}\right\rfloor+1}{\left|\psi\left(m^{\prime}\right)\right|}-\frac{N_{1}}{\left|\psi\left(N_{2}\right)\right|}$.

Thus for all $|w|>\left|\psi\left(N_{2}\right)\right|$, we also get $d\left(\psi\left(z_{k}\right), w\right)>1-3 \epsilon / 8$.

Step 3. Without loss of generality, we may assume that

$$
\frac{2 N_{1}+\left|\psi\left(N_{2}\right)\right|}{N_{4}}<\frac{\epsilon}{8}
$$


Assume that $w$ is a suffix of some $\psi\left(x_{j}\right)$. Let $j^{\prime}$ be the largest integer such that $\psi\left(j^{\prime}\right)$ intersects $w$. Then there is a prefix $r$ of $z_{j^{\prime}}$ such that $w$ is a suffix of $\psi\left(z_{j^{\prime}} j^{\prime} r\right)$. So we can write $w$ as either $w=w_{1} \psi\left(j^{\prime}\right) \psi(r)$ or $w=w_{1} \psi(r)$. If $|w|>N_{4}$, then at least one of the following inequalities holds:
(i) $\left|\psi\left(j^{\prime}\right)\right|>\left|\psi\left(N_{2}\right)\right|$,
(ii) $\left|w_{1}\right|>N_{1}$,
(iii) $\left|w_{2}\right|>N_{1}$.

Thus by Steps 1 and 2 , we also get $d\left(\psi\left(z_{k}\right), w\right)>1-\epsilon / 2$ for all $|w|>N_{4}$.

Step 4. Pick $N_{5}=N_{5}(\epsilon, k)$ which satisfies

$$
N_{5}>\left|\psi\left(N_{2}\right)\right|+2\left|\psi\left(z_{N_{2}}\right)\right| \text { and } \frac{2 N_{1}+\left|\psi\left(N_{2}\right)\right|+N_{4}}{N_{5}}<\frac{\epsilon}{8} .
$$

Now let $w$ be a subword of $\mathbf{u}$ of length $n>N_{5}(\epsilon, k)$. There are four cases:

CASE 1: $w=w_{1} \psi(j) w_{2}$, where $w_{1}$ is a suffix of $\psi\left(z_{j}\right)$ and $w_{2}$ is a prefix of $\psi\left(z_{j}\right)$. Since $n>N_{5}$, we have $n>\left|\psi\left(N_{2}\right)\right|+2\left|\psi\left(z_{N_{2}}\right)\right|, j \geq N_{2}$ and $2 N_{1} / n<\epsilon / 8$. Hence $d\left(\psi\left(z_{k}\right), w\right)>1-3 \epsilon / 8$ for all $|w|>N_{5}$.

CASE 2: $w$ is a subword of $\psi\left(j z_{j}\right)$ without being a factor of $\psi(j)$. Then $w=w_{1} w_{2}$, where $w_{1}$ is a suffix of $\psi(j)$ and $w_{2}$ is a prefix of $\psi\left(z_{j}\right)$. Since $n>N_{5}$, we have $n>\left|\psi\left(N_{2}\right)\right|+\left|\psi\left(z_{N_{2}}\right)\right|, j \geq N_{2}$ and $\left(N_{1}+\left|\psi\left(N_{2}\right)\right|\right) / n<$ $\epsilon / 8$. Thus $d\left(\psi\left(z_{k}\right), w\right)>1-\epsilon / 2$ for all $|w|>N_{5}$.

CASE 3: $w$ is a subword of $\psi\left(z_{j} j\right)$ without being a factor of $\psi(j)$. Then $w=w_{1} w_{2}$, where $w_{1}$ is a suffix of $\psi\left(z_{j}\right)$ and $w_{2}$ is a prefix of $\psi(j)$. By the argument of Case 2, when $n>N_{5}$, one has $d\left(\psi\left(z_{k}\right), w\right)>1-\epsilon / 2$.

CASE 4: $w$ is a factor of $\psi(j)$ where $j$ is the smallest integer such that $w$ appears in $\psi(j)$. Then $w$ can be written as $w=w_{1} e w_{2}$, where $w_{1}$ is a suffix of $\psi\left(x_{\lfloor\log j\rfloor-1}\right)$ and $w_{2}$ is a prefix of $\psi(\lfloor j / 2\rfloor)$. Since $n>N_{5}$, we have $\left(N_{4}+1+\left|\psi\left(N_{2}\right)\right|\right) / n<\epsilon / 8$. Thus $d\left(\psi\left(z_{k}\right), w\right)>1-7 \epsilon / 8$ for all $|w|>N_{5}$.

REMARK 3.5. In proving Lemma 3.4, for simplicity, we restrict our attention to the class of functions of the form $\left\{\varphi(t)=t^{\alpha}, 0<\alpha<1\right\}$. Indeed Lemma 3.4 still holds for the class of all functions which satisfy Cassaigne's conditions. Also the proof of Lemma 3.4 shows that the system $X_{\alpha}$ is of rank 1 with respect to the invariant measure $\mu_{\alpha}$.

Theorem 3.6. Each symbolic system $X_{\alpha}$ is uniquely ergodic.

Proof. Let $B$ be a block of length $p$. As in Proposition 3.1, let $f_{j}^{B}$ denote the frequency of the block $B$ in $\psi\left(z_{j}\right)$, and $f^{B}$ be the limit of the sequence $f_{j}^{B}$. By Theorem 3.2 we need to show that for any $x \in X_{\alpha}$,

$$
\lim _{n \rightarrow \infty} \frac{1}{n} \sum_{i=0}^{n-1} \mathbf{1}_{B} \circ \sigma^{i}(x)=\lim _{n \rightarrow \infty} \frac{1}{n} \sum_{i=0}^{n-1} \mathbf{1}_{B} \circ \sigma^{i}\left(\mathbf{u}_{\alpha}\right)=f^{B} .
$$

For given $\epsilon>0$, let $k$ and $N(\epsilon, k)$ be as in Lemma 3.4. Without loss of generality, we may assume that $\left|f_{k}^{B}-f^{B}\right|<\epsilon$ and $2 p /\left|\psi\left(z_{k}\right)\right|<\epsilon$. 
Since $x \in X_{\alpha}$, for each $n>N(\epsilon, k)$, there exists $m=m(n)$ such that $x_{0} x_{1} \cdots x_{n+p-2}=u_{m} u_{m+1} \cdots u_{m+n+p-2}$, which is a subword $w$ of $\mathbf{u}_{\alpha}$ and we have $d\left(\psi\left(z_{k}\right), w\right)>1-\epsilon$. Note that $\sum_{i=0}^{n-1} \mathbf{1}_{B} \circ \sigma^{i}(x)$ is equal to the number of occurrences of the given block $B$ in $w, d\left(\psi\left(z_{k}\right), w\right) \cdot(n+p-1) /\left|\psi\left(z_{k}\right)\right|$ is the maximum number of nonoverlapping occurrences of $\psi\left(z_{k}\right)$ in $w$, and $(n+p-1)-d\left(\psi\left(z_{k}\right), w\right) \cdot(n+p-1)$ is the number of positions of $w$ which are not covered by $\psi\left(z_{k}\right)$. Since $B$ can appear in the $\psi\left(z_{k}\right)$-covering part of $w$ or in the non- $\psi\left(z_{k}\right)$-covering part of $w$, or intersect both parts, the following inequalities hold:

$$
\begin{aligned}
\frac{d\left(\psi\left(z_{k}\right), w\right) \cdot(n+p-1)}{\left|\psi\left(z_{k}\right)\right|} \cdot f_{k}^{B} \cdot \mid & \psi\left(z_{k}\right) \mid \leq \sum_{i=0}^{n-1} \mathbf{1}_{B} \circ \sigma^{i}(x) \\
\leq & \frac{d\left(\psi\left(z_{k}\right), w\right) \cdot(n+p-1)}{\left|\psi\left(z_{k}\right)\right|} \cdot f_{k}^{B} \cdot\left|\psi\left(z_{k}\right)\right| \\
& +\left((n+p-1)-d\left(\psi\left(z_{k}\right), w\right) \cdot(n+p-1)\right) \\
& +\frac{d\left(\psi\left(z_{k}\right), w\right) \cdot(n+p-1)}{\left|\psi\left(z_{k}\right)\right|} \cdot 2 p .
\end{aligned}
$$

Hence we have

$$
\begin{aligned}
& \left|\frac{1}{n} \sum_{i=0}^{n-1} \mathbf{1}_{B} \circ \sigma^{i}(x)-f^{B}\right| \\
& \leq\left|f^{B}-f_{k}^{B}\right|+\left|f_{k}^{B}-\frac{n+p-1}{n} f_{k}^{B} d\left(\psi\left(z_{k}\right), w\right)\right|+\frac{n+p-1}{n}\left|1-d\left(\psi\left(z_{k}\right), w\right)\right| \\
& \quad+\frac{n+p-1}{n} d\left(\psi\left(z_{k}\right), w\right) \cdot \frac{2 p}{\left|\psi\left(z_{k}\right)\right|} \\
& <\epsilon+f_{k}^{B}\left|1-\frac{n+p-1}{n}(1-\epsilon)\right|+\frac{n+p-1}{n} \epsilon+\frac{n+p-1}{n} d\left(\psi\left(z_{k}\right), w\right) \epsilon<5 \epsilon .
\end{aligned}
$$

This completes the proof.

4. Entropy dimension of the measurable system $X_{\alpha}$. In this section, we will show that the entropy dimension of each measurable system $X_{\alpha}$ is zero. Let $\mathcal{P}$ be the generating partition of $\left(X_{\alpha}, \sigma\right)$ induced by the zero coordinate, i.e. $\mathcal{P}=\left\{P_{0}, P_{1}\right\}$ with $P_{0}=\left\{x \in X_{\sigma}: x_{0}=0\right\}$ and $P_{1}=\left\{x \in X_{\sigma}: x_{0}=1\right\}$.

Proposition 4.1. For any $\tau>0$,

$$
\lim _{n \rightarrow \infty} \frac{H_{\mu}\left(\bigvee_{i=0}^{n-1} \sigma^{-i} \mathcal{P}\right)}{n^{\tau}}=0
$$


Proof. Let $\mu$ be the unique invariant measure of $X_{\alpha}$. For any $n$-block $B$,

$$
\mu(B)=\lim _{m \rightarrow \infty} \frac{\sum_{i=g(n)+1}^{m-1} 2^{m-1-i} g(B, i)}{\sum_{i=0}^{m-1} 2^{m-1-i}|\psi(i)|}=\frac{\sum_{i=g(n)+1}^{\infty} 2^{-i} g(B, i)}{\sum_{i=0}^{\infty} 2^{-i}|\psi(i)|},
$$

where $g(B, i)$ is the number of occurrences of $B$ in $\psi\left(z_{i+1}\right)$ such that $B$ intersects $\psi(i)$, and $g(n)=\max \left\{i:\left|\psi\left(z_{i+1}\right)\right| \leq n-2\right\}$. Note that $g(B, i)=0$ for $i \leq g(n)$. Denoting $M=\sum_{i=0}^{\infty} 2^{-i}|\psi(i)|$, we have

$$
\begin{aligned}
H_{\mu}\left(\bigvee_{i=0}^{n-1} \sigma^{-i} \mathcal{P}\right) & =\sum_{|B|=n}-\mu(B) \log \mu(B) \\
& =\sum_{|B|=n}\left(-\sum_{i=g(n)+1}^{\infty} \frac{2^{-i} g(B, i)}{M}\right) \log \left(\sum_{i=g(n)+1}^{\infty} \frac{2^{-i} g(B, i)}{M}\right) \\
& \leq \sum_{i=g(n)+1}^{\infty} \sum_{|B|=n}-\frac{2^{-i} g(B, i)}{M} \log \frac{2^{-i} g(B, i)}{M} \\
& =\sum_{i=g(n)+1}^{\infty} \sum_{|B|=n} g(B, i) \frac{-2^{-i}}{M}\left(\log \frac{2^{-i}}{M}+\log g(B, i)\right) \\
& \leq \sum_{i=g(n)+1}^{\infty} \sum_{|B|=n} g(B, i) \frac{-2^{-i}}{M}\left(\log \frac{2^{-i}}{M}\right) .
\end{aligned}
$$

Since

$$
\sum_{\{B: n \text {-block }\}} g(B, i)=|\psi(i)|+n-1,
$$

we have

$$
\begin{aligned}
H_{\mu} & \left(\bigvee_{i=0}^{n-1} \sigma^{-i} \mathcal{P}\right) \leq \sum_{i=g(n)+1}^{\infty}(|\psi(i)|+n-1) \frac{-2^{-i}}{M}\left(\log \frac{2^{-i}}{M}\right) \\
& \leq \sum_{i=g(n)+1}^{\infty}(i+n-1) \frac{2^{-i}}{M}(i+\log M) \leq \sum_{i=g(n)+1}^{\infty}(i+n-1) \frac{2^{-i}}{M}(2 i) \\
& =\frac{2}{M}\left(\sum_{i=g(n)+1}^{\infty} i^{2} 2^{-i}+(n-1) \sum_{i=g(n)+1}^{\infty} i 2^{-i}\right) \\
& \leq \frac{2}{M}\left(\sum_{i=g(n)+1}^{\infty} i^{2} 2^{-i}+(n-1) \sum_{i=g(n)+1}^{\infty} i^{2} 2^{-i}\right)=\frac{2 n}{M} \sum_{i=g(n)+1}^{\infty} i^{2} 2^{-i} \\
& =\frac{2 n}{M}\left(4 \cdot 2^{-(g(n)+1)}(g(n)+1)+6 \cdot 2^{-(g(n)+1)}+2 \cdot 2^{-(g(n)+1)}(g(n)+1)^{2}\right) \\
& \leq \frac{24 n}{M} 2^{-(g(n)+1)}(g(n)+1)^{2} .
\end{aligned}
$$


Thus by Lemma 2.3, for any $\tau>0$, we have

$$
\lim _{n \rightarrow \infty} \frac{H_{\mu}\left(\bigvee_{i=0}^{n-1} \sigma^{-i} \mathcal{P}\right)}{n^{\tau}}=0
$$

The variational principle for entropy says that the topological entropy of a dynamical system $(X, T)$ is the maximum value of all measure-theoretic entropies of $T$. In the following, we will show that it is not the case for entropy dimension.

Proposition 4.2. For each dynamical system $\left(X_{\alpha}, \sigma\right)$, its entropy dimension is identically zero.

Proof. Let $\mathcal{P}$ be the generating partition induced by the zero coordinate on the system $X_{\alpha}$. We recall that $P_{[0, n)}(x)$ denotes the atom of $\bigvee_{i=0}^{n-1} \sigma^{-i} \mathcal{P}$ which $x$ belongs to. By Proposition 4.1, we have

$$
\lim _{n \rightarrow \infty} \frac{1}{n^{\tau}} \int_{X}-\log \mu\left(P_{[0, n)}(x)\right) d \mu(x)=0 .
$$

Hence for any $\epsilon>0$, if $n$ is large enough then

$$
\frac{1}{n^{\tau}} \int_{X}-\log \mu\left(P_{[0, n)}(x)\right) d \mu(x)<\epsilon .
$$

Thus the measure of the set $A=\left\{x:-\log \mu\left(P_{[0, n)}(x)\right)>n^{\tau}\right\}$ is less than $\epsilon$. So if $n$ is large enough, then $K(n, \epsilon)<\exp \left(n^{\tau}\right)$. This completes the proof.

TheOrem 4.3. For entropy dimension, the variational principle does not hold.

Proof. As in [DHP1, taking $\varphi(t)=t^{\alpha}$, we get an infinite word $\mathbf{u}_{\alpha}$ and the associated dynamical system $\left(X_{\alpha}, \sigma\right)$ whose topological entropy dimension is $\alpha$. By Theorem 3.6 and Proposition 4.2, $\left(X_{\alpha}, \sigma\right)$ is uniquely ergodic and its measure-theoretic entropy dimension is zero. This yields the result.

It is known that a measurable system conjugates to a group rotation if and only if there exist no sequences along which the measurable sequence entropy is positive (see $[\underline{K}]$ ). If a zero entropy system has enough "randomness", then we expect that there exists a sequence of integers along which the system exhibits positive sequence entropy. In the study of topological entropy dimension [DHP1], we introduced the following notion.

We say an increasing integer sequence $S=\left\{s_{1}<s_{2}<\cdots\right\} \subset \mathbb{Z}_{+}$is an entropy generating sequence of an open cover $\mathcal{U}$ if

$$
\liminf _{n \rightarrow \infty} \frac{1}{n} H\left(\bigvee_{i=1}^{n} T^{-s_{i}} \mathcal{U}\right)>0
$$


Another way of understanding the subexponential growth rate is to consider the "thickness" of these subsequences of $\mathbb{Z}^{+}$. To compute the "thickness", we define the dimension of a subset of $\mathbb{Z}^{+}$as follows.

DeFinition 4.4. Let $S=\left\{0 \leq s_{1}<s_{2}<\cdots\right\}$ be an increasing sequence of integers. For $\tau \geq 0$, we define

$$
\bar{D}(S, \tau)=\limsup _{n \rightarrow \infty} \frac{n}{s_{n}^{\tau}} \quad \text { and } \quad \underline{D}(S, \tau)=\liminf _{n \rightarrow \infty} \frac{n}{s_{n}^{\tau}} .
$$

We also define the upper dimension $\bar{D}(S)$ and the lower dimension $\underline{D}(S)$ of $S$ by

$$
\bar{D}(S)=\inf \{\tau \geq 0: \bar{D}(S, \tau)=0\} \quad \text { and } \quad \underline{D}(S)=\inf \{\tau \geq 0: \underline{D}(S, \tau)=0\} .
$$

If $\bar{D}(S)=\underline{D}(S)$, then it is called the dimension of the sequence $S$.

It is clear that if $S$ has positive density, then $\bar{D}(S)=\underline{D}(S)=1$. Also for example, if $S=\left\{n^{3}\right\}$, then $\bar{D}(S)=\underline{D}(S)=1 / 3$, and if $S=\left\{2^{n}\right\}$, then $\bar{D}(S)=\underline{D}(S)=0$. We note that the dimension differentiates the set $S=\left\{n^{3}\right\}$ from $S=\left\{n^{2}\right\}$ and $S=\left\{2^{n}\right\}$, although all of them have density 0 .

In [DHP1, DP], the authors have shown that the topological entropy dimension is related to the dimension of entropy generating sequences. In fact, it is shown that if a topological system $(X, T)$ has a finite generating open cover, then there exists an entropy generating sequence whose dimension is the same as the topological entropy dimension of $(X, T)$. The proof uses an idea of $[\mathrm{KL}$.

Let $(X, \mathcal{B}, \mu, T)$ be a measure preserving dynamical system and $\mathcal{P} \in P_{X}$ where $P_{X}$ is the collection of all measurable finite partitions of $X$. Analogous to the topological case, we say an increasing integer sequence $S=\left\{s_{1}<\right.$ $\left.s_{2}<\cdots\right\} \subset \mathbb{Z}_{+}$is an entropy generating sequence of a partition $\mathcal{P}$ if

$$
\liminf _{n \rightarrow \infty} \frac{1}{n} H_{\mu}\left(\bigvee_{i=1}^{n} T^{-s_{i}} \mathcal{P}\right)>0
$$

We define

$$
\bar{D}_{e}^{\mu}(T, \mathcal{P})= \begin{cases}\sup _{S \in \mathcal{E}_{\mu}(T, \mathcal{P})} \bar{D}(S) & \text { if } \mathcal{E}_{\mu}(T, \mathcal{P}) \neq \emptyset \\ 0 & \text { if } \mathcal{E}_{\mu}(T, \mathcal{P})=\emptyset\end{cases}
$$

where $\mathcal{E}_{\mu}(T, \mathcal{P})$ is the set of all entropy generating sequences of $\mathcal{P}$, and

$$
\bar{D}_{e}^{\mu}(X, T):=\sup _{\mathcal{P} \in P_{X}} \bar{D}_{e}^{\mu}(T, \mathcal{P}),
$$

which is called the dimension via entropy generating sequences of the system $(X, \mathcal{B}, \mu, T)$. It is known that if $\mathcal{P}$ is a generating partition of $X$, then $\bar{D}_{e}^{\mu}(X, T)=\bar{D}_{e}^{\mu}(T, \mathcal{P})[\mathrm{DHP} 2$. 
REMARK 4.5. We will show that the dimension via entropy generating sequences of each measurable system $X_{\alpha}$ is also zero.

Assume that $\bar{D}_{e}^{\mu}(T, \mathcal{P})>0$. Then there exist a positive real number $\tau$ and a sequence $S=\left\{s_{1}<s_{2}<\cdots\right\} \in \mathcal{E}_{\mu}(T, \mathcal{P})$ with $\bar{D}(S)>\tau$, i.e. $\lim \sup _{n \rightarrow \infty} n / s_{n}^{\tau}=\infty$. Since $S \in \mathcal{E}_{\mu}(T, \mathcal{P})$ there exists $c>0$ such that $H_{\mu}\left(\bigvee_{i=1}^{n} T^{-s_{i}} \mathcal{P}\right) / n>c$ for sufficiently large $n$. So we have

$$
\begin{aligned}
\limsup _{m \rightarrow \infty} \frac{H_{\mu}\left(\bigvee_{i=1}^{m} T^{-i} \mathcal{P}\right)}{m^{\tau}} & \geq \limsup _{n \rightarrow \infty} \frac{H_{\mu}\left(\bigvee_{i=1}^{s_{n}} T^{-i} \mathcal{P}\right)}{s_{n}^{\tau}} \\
& \geq \limsup _{n \rightarrow \infty} \frac{H_{\mu}\left(\bigvee_{i=1}^{n} T^{-s_{i}} \mathcal{P}\right)}{n} \cdot \frac{n}{s_{n}^{\tau}} \\
& \geq \limsup _{n \rightarrow \infty} c \cdot \frac{n}{s_{n}^{\tau}}=\infty
\end{aligned}
$$

This contradicts Proposition 4.1. Hence for each symbolic system $X_{\alpha}$, its dimension via entropy generating sequences is also identically zero.

Acknowledgements. The first author was partially supported by Weonil Foundation. The second author was partially supported by NNSF of China (grant no. 10901080). The second author would like to thank BK 21 program of Mathematics Department, Ajou University. The third author was partially supported by KRF (grant no. 2007-313-C00044).

\section{References}

[C] J. Cassaigne, Constructing infinite words of intermediate complexity, in: Developments in Language Theory, Lecture Notes in Comput. Sci. 2450, Springer, Berlin, 2003, 173-184.

[DHP1] D. Dou, W. Huang and K. K. Park, Entropy dimension of topological dynamical systems, Trans. Amer. Math. Soc., to appear.

[DHP2] - - - - Entropy dimension of measure-preserving systems, preprint.

[DP] D. Dou and K. Park, Examples of entropy generating sequences, preprint.

[F] S. Ferenczi, Measure-theoretic complexity of ergodic systems, Israel J. Math. 100 (1997), 189-207.

[FP] S. Ferenczi and K. K. Park, Entropy dimensions and a class of constructive examples, Discrete Contin. Dynam. Systems 17 (2007), 133-141.

[G] E. Glasner, Ergodic Theory via Joinings, Math. Surveys Monogr. 101, Amer. Math. Soc., 2003.

[KT] A. Katok and J.-P. Thouvenot, Slow entropy type invariants and smooth realization of commuting measure-preserving transformations, Ann. Inst. H. Poincaré Probab. Statist. 33 (1997), 323-338.

[KL] D. Kerr and H. Li, Independence in topological and $C^{*}$-dynamics, Math. Ann. 338 (2007), 869-926.

[K] A. G. Kushnirenko, On metric invariants of entropy type, Russian Math. Surveys 22 (1967), 53-61.

[R] D. Rudolph, Fundamentals of Measurable Dynamics, Clarendon Press, Oxford, 1990. 
[W] P. Walters, An Introduction to Ergodic Theory, Grad. Texts in Math. 79, Springer, New York, 1982.

[WS] S. Williams, Toeplitz minimal flows which are not uniquely ergodic, Z. Wahrsch. Verw. Gebiete 67 (1984), 95-107.

Young-Ho Ahn

Department of Mathematics

Mokpo National University

534-729 Mokpo, South Korea

E-mail:yhahn@mokpo.ac.kr

Kyewon Koh Park

Department of Mathematics

Ajou University

442-749 Suwon, South Korea

E-mail: kkpark@ajou.ac.kr
Dou Dou

Department of Mathematics

Nanjing University 210093 Nanjing, P.R. China E-mail: doumath@163.com

Received February 6, 2010

Revised version May 10, 2010 